The incidence of cancer and mortality in paediatric onset inflammatory bowel disease in Denmark and Finland during a 23-year period : a population-based study

\title{
Malham, Mikkel
}

2019-07

Malham , M , Jakobsen , C , Paerregaard , A , Virta , L J , Kolho , K-L \& Wewer , V 2019 , '

The incidence of cancer and mortality in paediatric onset inflammatory bowel disease in Denmark and Finland during a 23-year period : a population-based study ' , Alimentary Pharmacology \& Therapeutics , vol. 50 , no. 1 , pp. 33-39 . https://doi.org/10.1111/apt.15258

http://hdl.handle.net/10138/317191

https://doi.org/10.1111/apt.15258

acceptedVersion

Downloaded from Helda, University of Helsinki institutional repository.

This is an electronic reprint of the original article.

This reprint may differ from the original in pagination and typographic detail.

Please cite the original version. 


\title{
The incidence of cancer and mortality in paediatric onset inflammatory bowel disease in Denmark and Finland during a 23-year period: a population-based study
}

\author{
Mikkel Malham ${ }^{1}$ (D) | Christian Jakobsen ${ }^{1,2}$ | Anders Paerregaard ${ }^{1}$ | Lauri J. Virta ${ }^{3}$ | \\ Kaija-Leena Kolho ${ }^{4,5}$ (D) | Vibeke Wewer ${ }^{1}$
}

${ }^{1}$ The Paediatric Department, Copenhagen University Hospital, Hvidovre, Denmark

${ }^{2}$ The Gastro Unit, Medical Division, Copenhagen University Hospital, Hvidovre, Denmark

${ }^{3}$ The Research Department, The Social Insurance Institution, Turku, Finland

${ }^{4}$ The Paediatric Department, Tampere University Hospital and Tampere University, Tampere, Finland

${ }^{5}$ Faculty of Medicine, University of Helsinki, Helsinki, Finland

\section{Correspondence}

Dr. Mikkel Malham, The Paediatric

Department, Copenhagen University Hospital, Hvidovre, Denmark.

Email: mikkel.malham.knudsen.01@regionh. $\mathrm{dk}$

Funding information

This study was in part funded by ESPGHAN's Network grant, Nordic Cancer Union, Arvid Nilsson's Foundation, Copenhagen University's Foundation for Cancer Research, AP Møller Foundation and Højmosegaard. None of the funding authorities have had any influence on study design, results or drafting off the final paper.

\section{Summary}

Background: Recent studies report increased risks of both cancer and mortality in paediatric onset inflammatory bowel disease (pIBD) but the reproducibility of this is unknown.

Aim: In this population-based study we aim to estimate the risk of cancer and mortality in the Danish and Finnish pIBD population in a 23-year period compared to the general population.

Methods: The pIBD population was defined as individuals registered in the national patient registries with a diagnosis of Crohn's disease (CD), ulcerative colitis (UC) or IBD-unclassified before their 18th birthday from 1992 to 2014. This cohort was cross referenced with the national cancer and mortality registries identifying all pIBD patients who subsequently developed cancer and/ or died and followed up to the end of 2014. Risk estimates are presented as standardised incidence ratios calculated based on incidence figures from the populations.

Results: Six thousand six hundred and eight-nine patients with pIBD were identified (median age at follow-up 22.3 years; median follow-up: 9.6 years [interquartile range: 4.8-16.0]). Seventy-two subsequently developed cancer and 65 died. The standardised incidence ratio of cancer in general was $2.6(95 \% \mathrm{Cl}: 1.8-3.7)$ and $2.5(95 \% \mathrm{Cl}$ : 1.8-3.4) in CD and UC, respectively. The standardised mortality ratios were 2.2 (95\% $\mathrm{Cl}$ : 1.4-3.4) and $3.7(95 \% \mathrm{Cl}$ : 2.7-5.0) in $\mathrm{CD}$ and $\mathrm{UC}$, respectively. The leading causes for mortality were cancer, suicide and infections.

Conclusions: We found an increased risk of cancer and mortality in pIBD. This underlines the importance of cancer surveillance programs and assessment of mental health in the standard of care in adolescent pIBD patients.

Kaija-Leena Kolho and Vibeke Wewer should be considered joint senior author. 


\section{1 | INTRODUCTION}

The incidence of cancer and mortality in IBD has been extensively debated for decades. Recent results from studies reporting on colorectal cancers (CRC), also termed disease associated cancers, are inconsistent with the historical literature, reporting lower risk estimates and some finding no increased risk of cancer. ${ }^{1-4}$ The reasons for this decline in incidence is unknown, but is speculated to be due to new treatment modalities and colectomies performed at an earlier stage. ${ }^{5,6}$

The few population-based cancer studies that include subanalyses on paediatric onset IBD ( $P$ IBD) have found an overall two-three fold increased risk of cancer. ${ }^{2,7,8}$ In the paediatric literature on malignancy in IBD, only two population-based studies exist. One of these reported a 25 -fold increase in CRC in paediatric onset ulcerative colitis ( $\mathrm{UUC}$ ), but this study included only 69 patients of whom two developed CRC. ${ }^{9}$ The last and largest study in PIBD, a nation-wide study from Sweden, demonstrated a twofold increase in cancer in general and a 18-fold increase in gastro-intestinal cancers. ${ }^{10}$

Regarding mortality, the risk of death in IBD seems to be declining, especially in UC. However, compared to the general population, there is an increased risk of mortality in both CD and UC. ${ }^{11}$ In pIBD knowledge is sparse regarding the risk and causes of mortality. Joosse et al published a 3-year prospective study in 2018 from multiple centres in Europe. ${ }^{12}$ They reported 26 deaths with the main causes of mortality being cancer and infections. Later in 2018 , Olén et al published a nation-wide study from Sweden, which is to date the largest study regarding this topic. ${ }^{13}$ They found a fourfold increase in the risk of mortality in pUC and a twofold risk in paediatric onset $C D(p C D)$ with the main cause of mortality being cancer.

Based on these observations it seems plausible that pIBD is an important risk factor of both cancer and mortality in both late adolescent and early adulthood. However, our knowledge on this subject is primarily derived from studies performed in a single study cohort (the two nationwide Swedish studies ${ }^{10,13}$ ). Hence, we aim to estimate the risk of cancer and mortality in pIBD diagnosed in two more recent nationwide population-based cohorts from Denmark and Finland.

\section{MATERIALS AND METHODS}

\section{1 | Study design}

In this population-based retrospective registry study we included all patients diagnosed with pIBD in Denmark and Finland in the period from 1 January 1992 to 31 December 2014. PIBD was defined as patients who were diagnosed with IBD before their 18th birthday. The pIBD cohort was crosslinked (through a unique social security number given to all citizens in both Denmark and Finland) to the national cancer registries and the national cause of death registries, where patients who developed cancer (any) or died after their IBD diagnosis were identified.

\section{2 | Study population}

In both Danish and Finnish registries, IBD was defined as International Classification of Diseases eight revision (ICD-8) codes 56300-56309, ninth revision (ICD-9) code 555 or tenth revision (ICD-10) code group K50 or ICD-8 code 56319 , ICD-9 code 556 or ICD-10 code group $\mathrm{K} 51$.

In Denmark, the pIBD cohort was identified through the Danish National Patient Registry (LPR), which contains information of inpatient hospitalisation from 1977 and outpatient contacts from 1994. We considered the diagnosis as valid if two or more entries were made with an IBD diagnosis, as this has previously been found to ensure the validity. ${ }^{14}$ Data regarding surgery (colectomy) were also collected through the LPR. The Danish pIBD cohort was crosslinked to the Danish Cancer Registry and Causes of Death Registry. The Danish Cancer Registry contains data on all patients diagnosed with any cancer in Denmark (including exact diagnosis and date of diagnosis). The Causes of Death Registry contains data on cause of and time of death. To verify both the IBD and the cancer diagnosis, we crosslinked cancer cases and deaths with the Danish Pathology Database.

In Finland, the pIBD cohort was identified through the Special Reimbursement Registry which is maintained by the Social Insurance Institution of Finland. This registry includes data on diagnosis of all patients eligible for special reimbursement of medical expenses, including IBD. This registry has previously been shown to have an excellent coverage and validity and has been used to estimate the incidence of pIBD in Finland. ${ }^{15-17}$ The pIBD cohort was crosslinked to the National Institute for Health and Welfare (THL), the Cancer Registry and the Causes of Death Registry. The Cancer Registry contains data on all patients diagnosed with any cancer in Finland (including exact diagnosis and date of diagnosis). ${ }^{18,19}$ The Causes of Death Registry are maintained by Statistics Finland and contains data on the cause of and time of death. To assess time at risk of colorectal cancer the pIBD cohort was crosslinked with the Care Registry for Health Care (HILMO), which contains data on all surgical interventions in Finland.

In both countries, we used the last recorded diagnosis code in the registries as the valid diagnosis. The age-specific cancer rates in the general population for each country were derived from the NORDCAN homepage, where cancer incidences in the Nordic countries are published. ${ }^{20}$ In both Denmark and Finland, the age-specific and sex specific number of deaths in each calendar year are accessible online (www.dst.dk and www.stat.fi).

\section{3 | Statistical analysis}

For cancer and mortality analyses, the number of person-years of follow-up was calculated from the date of IBD diagnosis to cancer diagnosis or death on 31 December 2014, whichever event came first. When estimating the risk of CRC, only person-years before colectomy were considered time at risk. 
The overall differences in cancer and mortality risks between the pIBD cohorts and the general population were calculated using standardised incidence ratios (SIR) and standardised mortality ratios (SMR). Among the pIBD patients, the numbers of observed cases and person-years at risk were calculated by sex and 5 -year age groups. Analyses were also performed separately for $C D$ and UC. The expected numbers of cases for total cancers, specific cancer sites, all-cause mortality and cause-specific mortality were calculated by multiplying the person-years at risk by the incidence rates of each cancer/ mortality in the respective sex, age and calendar period in the background population of the respective countries. To calculate SIR or SMR, the observed number of cases was divided by the expected number. For SIR and SMR, the exact $95 \%$ confidence intervals $(\mathrm{Cl})$ were calculated by assuming that the number of observed cancer and mortality cases followed a Poisson's distribution.

Statistical analyses were performed using SAS Enterprise (version 7.11, SAS institute Inc, Cary. NC. USA). The study was approved by the Danish Patient Safety Authority. As the study did not involve direct patient contact ethical approval is not applicable according to legislation in neither Denmark nor Finland.

\section{3 | RESULTS}

From 1992 to 2015 we identified 6,689 patients with pIBD. Of these, 3333 where identified in Denmark and 3357 were identified in Finland. The median age at IBD diagnosis was 14.0 years (Interquartile range (IQR): 12-16) and the median follow-up time was 9.6 years IQR 4.8-16.0. The background population in the correspondent age groups was 5416763 (2 517198 and 2899565 in Denmark and Finland, respectively). The baseline demographics are presented in Table 1 with the country specific demographics presented in Table S1. Total follow-up time of the pIBD cohort was 71675 person-years, with 37830 person-years in Denmark

TAB LE 1 Baseline characteristics (including cancer and mortality cases) in a Danish and Finnish paediatric IBD cohort diagnosed 1992-2014 and followed up to the end of 2014

\begin{tabular}{|llll|}
\hline & IBD-total & CD & UC \\
\hline Patients (n) & & & \\
\hline Total & 6689 & 2921 & 3741 \\
\hline Male & 3544 & 1648 & 1882 \\
\hline Female & 3145 & 1273 & 1859 \\
\hline Median age at IBD & $14.0(12-16)$ & $14.0(12-16)$ & $14.0(11-16)$ \\
\hline diagnosis (y [IQR]) & & & \\
\hline Median follow-up & $9.6(4.8-16.0)$ & 9.5 & 9.8 \\
time (y [IQR]) & & $(4.8-16.0)$ & $(4.8-16.0)$ \\
\hline Cancer cases (n) & 72 & 33 & 39 \\
\hline Deaths (n) & 65 & 21 & 44 \\
\hline
\end{tabular}

$\mathrm{CD}$, Crohn's disease; IBD, inflammatory bowel disease; IQR, interquartile range; UC, ulcerative colitis.
(18 467 CD/ 19284 UC/ 79 IBDU) and 33845 person-years in Finland (12 746 CD/ 20860 UC/ 239 IBDU). When combining the two cohorts, the median age at end of follow-up was 22.3 years (IQR:17.4-28.4) (CD 22.4 years [IQR: 17.4-28.4] and UC 22.2 years [IQR: 17.8-28.4]). In the combined pIBD cohort, 720 patients had a total colectomy performed with a median time from diagnosis to colectomy of 2.8 years (IQR: 1.0-6.1). A total of 1,365 patients (20.4\%) underwent bowel resection (including colectomy) during follow-up (median time from diagnosis to resection 2.6 years [IQR: 0.7-6.1]).

\subsection{Cancer}

Seventy-two cancer cases were observed in the pIBD cohort (38 and 34 in Denmark and Finland, respectively) with an incidence rate of 10/ 10000 person-years and a SIR of 2.5 (95\% CI: 2.0-3.2). The characteristics of the cancer cases are presented in Table 2 and the country specific characteristics are presented in Table S2. Median age at cancer diagnosis was 25.0 years (range: $13-35)$. The risk of cancer (any) was 9.0/ $10000,10.6 / 10000,34.7 / 10000$ and 75.8/ 10000 patients after 1, 5, 10 and 20 years of follow-up, respectively.

In CD, the overall cancer incidence was $10.7 / 10000$ personyears and the SIR was $2.6(\mathrm{Cl}: 1.8-3.7)$. The country specific incidence rates were 12.5/ 10000 and 7.8/10000 patient years in Denmark and Finland, respectively. The median time from IBD diagnosis to cancer was 11.2 years (range: 0.04-19.3).

In UC, the overall cancer incidence was 9.7/ 10000 patient years and the SIR was 2.5 (Cl: 1.8-3.4). The country specific incidence rates

TAB LE 2 Number and age of cancer and mortality cases in a Danish and Finnish paediatric IBD cohort diagnosed 1992-2014 and followed up to the end of 2014

\begin{tabular}{|c|c|c|c|}
\hline & IBD-total & $C D$ & UC \\
\hline \multicolumn{4}{|l|}{ Cancer } \\
\hline \multicolumn{4}{|l|}{ Patients (n) } \\
\hline Total & 72 & 33 & 39 \\
\hline Male & 37 & 15 & 22 \\
\hline Female & 35 & 18 & 17 \\
\hline $\begin{array}{l}\text { Median age at } \\
\text { cancer } \\
\text { diagnosis } \\
\text { (y [range]) }\end{array}$ & $25.0(13-35)$ & $24.0(15-35)$ & $25.0(13-33)$ \\
\hline \multicolumn{4}{|l|}{ Mortality } \\
\hline \multicolumn{4}{|l|}{ Patients (n) } \\
\hline Total & 65 & 21 & 44 \\
\hline Male & 39 & 16 & 23 \\
\hline Female & 26 & 5 & 21 \\
\hline $\begin{array}{l}\text { Median age at } \\
\text { mortality } \\
\text { (y [range]) }\end{array}$ & $22.0(8-36)$ & $24.0(8-36)$ & $22.0(14-34)$ \\
\hline
\end{tabular}

$\mathrm{CD}$, Crohn's disease; IBD, inflammatory bowel disease; UC, ulcerative colitis. 
TAB LE 3 Standardised incidence ratios of the main cancer groups and the number of specific cancer diagnoses in a Danish and Finnish paediatric IBD cohort diagnosed 1992-2014 and followed up to the end of 2014

\begin{tabular}{|c|c|c|c|}
\hline & IBD-total & $C D$ & UC \\
\hline All & $2.5(2.0-3.2)$ & $2.6(1.8-3.7)$ & $2.5(1.8-3.4)$ \\
\hline Colorectal cancers & $15.3(8.7-24.8)$ & $8.4(2.3-21.4)$ & $21.4(11.0-37.4)$ \\
\hline Small bowel cancers & $21.8(4.4-63.6)$ & $32.6(3.7-117.8)$ & $13.3(0.2-74)$ \\
\hline Liver cancers & $42.8(19.5-81.3)$ & $21.8(2.5-78.9)$ & $59.5(23.8-122.6)$ \\
\hline Skin cancers & $4.2(2.4-6.7)$ & $6.7(3.4-11.6)$ & $2.2(0.7-5.2)$ \\
\hline \multicolumn{4}{|l|}{ Number of cancer cases ( $n$ ) } \\
\hline \multicolumn{4}{|l|}{ Gastrointestinal } \\
\hline Colorectal cancer & 16 & 4 & 12 \\
\hline Hepatocellular carcinoma & 1 & 1 & 0 \\
\hline \multicolumn{4}{|l|}{ Lymphomas } \\
\hline Hodgkin's lymphoma & 7 & 4 & 3 \\
\hline Non-Hodgkin's lymphoma & 3 & 1 & 2 \\
\hline \multicolumn{4}{|l|}{ Skin cancer } \\
\hline Malignant melanoma & 10 & 7 & 3 \\
\hline Basal cell carcinoma & 7 & 5 & 2 \\
\hline \multicolumn{4}{|l|}{ Non-IBD related cancers } \\
\hline Genital cancers & 6 & 4 & 2 \\
\hline
\end{tabular}

$\mathrm{CD}$, Crohn's disease; $\mathrm{Cl}$, confidence interval; IBD, inflammatory bowel disease; UC, ulcerative colitis.

were 7.8/ 10000 and 11.5/ 10000 patient years in Denmark and Finland, respectively. The cancer specific SIRs are presented in Table 3 and the country specific SIRs are presented in Table S3. The median time from IBD diagnosis to cancer was 11.5 years (range: 0.06-21.2).

\section{2 | Mortality}

Of the patients in the pIBD cohort a total of 65 patients died, 29 in Denmark and 36 in Finland. The characteristics of the mortality cases are presented in Table 2 with the country specific characteristics presented in Table S2. The incidence rate of mortality was 9.1/ 10000 patient years, with an incidence rate of $7.7 / 10000$ patient years in Denmark and 10.6/ 10000 patient years in Finland. The overall (all cause) SMR was 3.0 ( $\mathrm{Cl}: 2.4-3.9)$ and the cause specific mortalities are presented in Table 4 with the country specific mortalities presented in Table S4. The median time from diagnosis to mortality was 9.4 years (range: 0.01-22.4) and the median age at mortality was 22.0 years (range: $8-35$ ). The leading causes of mortality were cancer (one in 4008 patient years), suicide (one in 4509 patient years) and infections (one in 6558 patient years). As neither Denmark nor Finland have age matched mortality rates available on the exact same causes of deaths as found in our study (except suicide), only suicide SMR can be calculated, which is presented in Table 4. Of the 12 patients who died due to infections, six patients had a sepsis diagnosis as the primary cause of death. Of these, one had a central venous catheter infection, one had peritonitis from a spontaneous perforated colon, one had toxic mega-colon, one had streptococcal sepsis and two had sepsis of unknown origin. The remaining six patients died from pneumonia $(n=5)$ and meningococcal meningitis $(n=1)$.

\section{DISCUSSION}

In this nationwide population-based study from Denmark and Finland, we followed pIBD patients from IBD diagnosis to cancer 
TAB LE 4 Standardised mortality ratios and the number of specific mortality causes in a Danish and Finnish paediatric IBD cohort diagnosed 1992-2014 and followed up to the end of 2014

\begin{tabular}{|c|c|c|c|}
\hline & IBD-total & $C D$ & UC \\
\hline \multicolumn{4}{|c|}{ Standardised mortality ratios $(95 \% \mathrm{Cl})$} \\
\hline All & $3.0(2.4-3.9)$ & $2.2(1.4-3.4)$ & $3.7(2.7-5.0)$ \\
\hline Suicide & $3.8(2.2-6.0)$ & $2.8(1.0-6.2)$ & $4.6(2.4-8.0)$ \\
\hline \multicolumn{4}{|c|}{ Cause specific mortality (n) } \\
\hline Cancer & 18 & 3 & 15 \\
\hline Infections & 11 & 3 & 8 \\
\hline Suicide & 18 & 6 & 12 \\
\hline Gastrointestinal & 2 & 2 & 0 \\
\hline Other & 16 & 7 & 9 \\
\hline
\end{tabular}

$\mathrm{CD}$, Crohn's disease; $\mathrm{Cl}$, confidence interval; IBD, inflammatory bowel disease; UC, ulcerative colitis.

diagnosis or mortality and found an increased risk of both cancer and mortality in patients with paediatric onset of IBD compared to the general population. The increased risk of cancer was mainly due to an increased frequency of colorectal adenocarcinomas (CRC), liver cancers, skin cancers and lymphomas and the median age of cancer diagnosis was 25 years. The three most common causes of death were cancer, suicide and infections and the median age of mortality was 22 years.

We found a 2.5 -fold increase in the risk of cancer in pIBD compared to the general population with identical risk estimates in $C D$ and UC. This is almost identical to the increased risk estimate found in the recent nationwide Swedish pIBD study ${ }^{10}$ and twice the risk estimate reported in the recent adult literature. ${ }^{7,21}$ In the Swedish study they, however, reported an almost 20 -fold increased risk of developing CRC which is higher than the 15 -fold increased risk reported in our study. Most studies reporting on CRC in pIBD have reported higher risk estimates of CRC than we found. ${ }^{1,9,21,22}$ Many of the previous studies, including the Swedish study, included historical patient cohorts dating back before the 1980s. As some adult studies have shown a decreasing risk of CRC over the last decades, ${ }^{1,4}$ we believe that the results presented in this paper more accurately reflects the actual risk of developing CRC for pIBD patients diagnosed within the past 10-20 years which is seven times the risk estimate of CRC in the adult population. ${ }^{2}$ We also found a very high risk of liver cancer, mainly cholangiocarcinomas in UC patients. In a primary sclerosing cholangitis (PSC) cohort from Sweden, in which $79 \%$ had IBD, the risk of cholangiocarcinoma was reported to be 160 -fold increased, compared with the general population. ${ }^{23}$ It could therefore be hypothesised, that the increased risk of cholangiocarcinoma reported in our study can be attributed solely to PSC. This study was not designed to evaluate concomitant PSC. However, in the Danish population, the data extract did allow for a post hoc analysis of concomitant PSC. A PSC diagnosis was only found in one of the six patients developing cholangiocarcinoma. Hence, it seems that the pIBD diagnosis increases the risk of liver cancer.
We found three cases of small bowel carcinomas (SBC) in the Finnish population (age at cancer diagnosis: 22-33 years. Year of diagnosis: 2003-2014). The risk of SBC has also been found to be increased in coeliac disease, ${ }^{24}$ which may be associated with IBD. Hence, it remains unknown if the increased SIR of SBC is explained by pIBD. The fact, that one of the three SBC's developed in a UC patient, who should have no inflammation in the short bowels, supports the hypothesis that the SBC might also depend on other factors than pIBD.

Due to the immunosuppressant effects of both immunomodulators and biologicals, IBD patients are considered to be at an increased risk of lymphoproliferative diseases (primarily lymphomas). ${ }^{25}$ Two tertiary centre studies found that pIBD patients had an increased risk lymphoproliferative diseases when treated with thiopurines. ${ }^{26,27}$ The only population-based study report on lymphoproliferative diseases in pIBD found a 2.7 times increased risk of developing lymphoproliferative diseases, ${ }^{10}$ which is similar to the risk estimates reported in our study. When stratifying by IBD diagnosis, only CD had a significantly increased risk with a SIR of 3.2. As thiopurines are standard treatment of pCD in Denmark and Finland and have been so since the late $1990 \mathrm{~s},{ }^{28}$ it is likely that the CD patients affected had been treated with thiopurines. However, due to the design of this study, we cannot elaborate on the risk factor of thiopurines in developing lymphoproliferative diseases (or any other cancer).

An increased risk of skin cancer has previously been reported in IBD. Our risk estimate is in line with the Swedish nationwide study ${ }^{10}$ but significantly higher than the estimates previous reported in adult IBD cohorts. ${ }^{7,21}$ In our study, the increased risk of skin cancer was restricted to CD patients (SIR 6.7), which was also found in the previous studies. Skin cancers, especially nonmelanoma, have been attributed to thiopurine treatment. A recent meta-analysis, including mainly adult studies, found that thiopurine treatment increased the risk of nonmelanoma skin cancer by $2.8 .^{29}$ However, as with lymphoproliferative disease, our study design does not allow further elaboration of treatment exposures effect on cancer risk.

We found a threefold increase in the standardised mortality ratio, with the peak incidence rate in UC (SMR 3.7), which is a threefold increase compared to results published in the aIBD population. ${ }^{11}$ This is in line with a recent nationwide study from Sweden ${ }^{13}$ were the main mortality causes (by number of cases) were cancer, gastrointestinal causes and trauma. Unexpectedly, in both Denmark and Finland, suicide was among the leading causes of mortality in our study. This was not reported in the Swedish study and may reflect a limitation in registry-based studies. We found the death rate due to suicide increased (SMR 4.0) compared to the general population. This has not previously been shown, but it may be due to the decreased quality of life and increased risk of depressive disorders in pIBD previously described. ${ }^{30,31}$

The other important cause of mortality (besides cancer) in our study, was infections. This is in line with two previous European multicentre studies and a systematic review reporting on cancer and mortality in pIBD. ${ }^{12,32,33}$ However, these studies where mainly 
case based and the systematic review is primarily based on those two studies.

The strength of this study is the nationwide design with inclusion of recent pIBD patients in both Denmark and Finland, which resulted in a sufficient sample size without including historical patients. The national patient registries have previously been shown to have excellent coverage. ${ }^{14-16}$ In Denmark, the LPR did not include outpatients until 1994. However, patients diagnosed with pIBD in Denmark are almost exclusively diagnosed by endoscopy, which is an inpatient procedure (included in the LPR since 1977), and pIBD patients, given its aggressive nature, ${ }^{34,35}$ will require long-term care in the outpatients clinic and, hence, also be included in the LPR before 1992. In Denmark, all cancer cases were verified through the national pathology database, which, by law, includes pathology reports from all cancer patients in Denmark. In Finland, the cancer registry has previously been shown to have an excellent coverage with over $98 \%$ of all cancers. ${ }^{18,19}$ As the hospitals in both Denmark and Finland are mainly government driven and all major expenses to medicine is deductible, the fact that this study included patients from both Denmark and Finland is not perceived as a problem by the authors, as patients in both countries had similar access to both immunomodulators, biologicals and surgery, if needed. Despite the previously proven validity of the Danish and Finnish registries, the main weakness of the present study is the retrospective nature of the study and the fact that we relied solely on registry data with no possibility to verify the data in patient charts. However, with the previously proven excellent coverage, we do not believe that this has flawed the reported results in this study. Lastly, the registries did not allow us to elaborate on disease severity, disease localisation or treatment exposures as risk factors of cancer or mortality.

In conclusion, we found an increased risk of both cancer and mortality in pIBD with cancer presentation from the late teens and early adulthood. This adds to the mounting evidence ${ }^{34-36}$ that pIBD is a distinct IBD phenotype and should be appreciated as a disease modifier predicting a more aggressive disease course compared to adult onset IBD. Moreover, the number of suicides was alarming, warranting attention to the mental health of the patients. Lastly, this study highlights the need for future studies designed to estimate possible risk factors of cancer development in pIBD. This would facilitate the design of an evidence-based cancer surveillance program in pIBD patients which, in the light of the present findings, appears to be needed in the standard care of adolescent pIBD patients.

\section{ACKNOWLEDGEMENTS}

The authors thank the following grants for financial support: ESPGHAN's Network grant, Nordic Cancer Union, Arvid Nilsson's Foundation, Copenhagen University's Foundation for Cancer Research, A.P. Moeller Foundation, Hoejmosegaard grant. The authors also thank MSc in statistics Thomas Kallemose for statistical help.

Declaration of personal interests: None.

\section{AUTHORSHIP}

Guarantor of the article: Vibeke Wewer.

Author contributions: All authors contributed to study conception, design and critical revision of the final manuscript for important intellectual content. Mikkel Malham contributed with data extraction from Danish registries and Kaija-Leena Kolho and Lauri J. Virta with data extraction from Finnish registries. Mikkel Malham analysed data and drafted the manuscript. All authors approved the final version of the article, including the authorship list.

\section{ORCID}

Mikkel Malham (iD https://orcid.org/0000-0002-6956-7676

Kaija-Leena Kolho (iD https://orcid.org/0000-0001-9170-8415

\section{REFERENCES}

1. Jess T, Simonsen J, Jorgensen KT, Pedersen BV, Nielsen NM, Frisch M. Decreasing risk of colorectal cancer in patients with inflammatory bowel disease over 30 years. Gastroenterology. 2012;143:374-375.

2. Jess T, Rungoe C, Peyrin-Biroulet L. Risk of colorectal cancer in patients with ulcerative colitis: a meta-analysis of population-based cohort studies. Clin Gastroenterol Hepatol. 2012;10: 639-645.

3. Söderlund S, Brandt L, Lapidus A, et al. Decreasing time-trends of colorectal cancer in a large cohort of patients with inflammatory bowel disease. Gastroenterology. 2009;136:1561-1569.

4. Castaño-Milla C, Chaparro M, Gisbert JP. Systematic review with meta-analysis: the declining risk of colorectal cancer in ulcerative colitis. Aliment Pharmacol Ther. 2014;39:645-659.

5. Sebastian S, Hernández V, Myrelid P, et al. Colorectal cancer in inflammatory bowel disease: results of the 3rd ECCO pathogenesis scientific workshop (I). J Crohns Colitis. 2014;8:5-18.

6. Carrat F, Seksik P, Colombel J-F, Peyrin-Biroulet L, Beaugerie L. The effects of aminosalicylates or thiopurines on the risk of colorectal cancer in inflammatory bowel disease. Aliment Pharmacol Ther. 2017:45:533-541.

7. Kappelman MD, Farkas DK, Long MD, et al. Risk of cancer in patients with inflammatory bowel diseases: a nationwide population-based cohort study with 30 years of follow-up evaluation. Clin Gastroenterol Hepatol. 2014;12:265-273.e1.

8. Ekbom A, Helmick C, Zack M, Adami HO. Ulcerative colitis and colorectal cancer. A population-based study. N Engl J Med. 1990;323:1228-1233.

9. Jakobsen C, Paerregaard A, Munkholm P, Wewer V. Paediatric inflammatory bowel disease during a 44-year period in Copenhagen County: occurrence, course and prognosis - a population-based study from the Danish Crohn Colitis Database. Eur J Gastroenterol Hepatol. 2009;21:1291-1301.

10. Olén O, Askling J, Sachs M, et al. Childhood onset inflammatory bowel disease and risk of cancer: a Swedish nationwide cohort study 1964-2014. BMJ. 2017;j3951:1964-2014.

11. Jess T, Frisch M, Simonsen J. Trends in overall and cause-specific mortality among patients with inflammatory bowel disease from 1982 to 2010. Clin Gastroenterol Hepatol. 2013;11:43-48.

12. Joosse ME, Aardoom MA, Kemos $P$, et al. Malignancy and mortality in paediatric-onset inflammatory bowel disease: a 3-year prospective, multinational study from the paediatric IBD Porto group of ESPGHAN. Aliment Pharmacol Ther. 2018:48:523-537. 
13. Olen $\mathrm{O}$, Askling J, Sachs MC, et al. Increased mortality of patients with childhood-onset inflammatory bowel diseases, compared with the general population. Gastroenterology. 2018;156:614-622.

14. Lophaven SN, Lynge E, Burisch J. The incidence of inflammatory bowel disease in Denmark 1980-2013: a nationwide cohort study. Aliment Pharmacol Ther. 2017;45:961-972.

15. Virta LJ, Saarinen MM, Kolho K-L. Inflammatory bowel disease incidence is on the continuous rise among all paediatric patients except for the very young: a nationwide registry-based study on 28-year follow-up. J Crohns Colitis. 2017;11:150-156.

16. Lehtinen $\mathrm{P}$, Ashorn $\mathrm{M}$, Iltanen $\mathrm{S}$, et al. Incidence trends of pediatric inflammatory bowel disease in Finland, 1987-2003, a nationwide study. Inflamm Bowel Dis. 2011;17:1778-1783.

17. Virta L, Auvinen A, Helenius H, Huovinen P, Kolho K-L. Association of repeated exposure to antibiotics with the development of pediatric Crohn's disease-a nationwide, register-based Finnish casecontrol study. Am J Epidemiol. 2012;175:775-784.

18. Korhonen P, Malila N, Pukkala E, Teppo L, Albanes D, Virtamo J. The Finnish Cancer Registry as follow-up source of a large trial cohortaccuracy and delay. Acta Oncol. 2002;41:381-388.

19. Teppo L, Pukkala E, Lehtonen M. Data quality and quality control of a population-based cancer registry. Experience in Finland. Acta Oncol. 1994;33:365-369.

20. Engholm G, Ferlay J, Christensen N, et al. NORDCAN: Cancer Incidence, Mortality, Prevalence and Survival in the Nordic Countries, Version 8.1 (28.06.2018). Association of the Nordic Cancer Registries. http://www-dep.iarc.fr/NORDCAN.htm. Published 2018. Accessed January 30, 2019.

21. Jess T, Horváth-Puhó E, Fallingborg J, Rasmussen $\mathrm{HH}$, Jacobsen BA. Cancer risk in inflammatory bowel disease according to patient phenotype and treatment: a Danish population-based cohort study. Am J Gastroenterol. 2013;108:1869-1876.

22. Weedon DD, Shorter RG, Ilstrup DM, Huizenga KA, Taylor WF. Crohn's disease and cancer. N Engl J Med. 1973;289:1099-1103.

23. Bergquist A, Ekbom A, Olsson R, et al. Hepatic and extrahepatic malignancies in primary sclerosing cholangitis. J Hepatol. 2002;36:321-327.

24. Ilus T, Kaukinen K, Virta LJ, Pukkala E, Collin P. Incidence of malignancies in diagnosed celiac patients: a population-based estimate. Am J Gastroenterol. 2014;109:1471-1477.

25. Smith MA, Irving PM, Marinaki AM, Sanderson JD. Review article: malignancy on thiopurine treatment with special reference to inflammatory bowel disease. Aliment Pharmacol Ther. 2010;32:119-130.

26. Hyams JS, Dubinsky MC, Baldassano RN, et al. Infliximab is not associated with increased risk of malignancy or hemophagocytic lymphohistiocytosis in pediatric patients with inflammatory bowel disease. Gastroenterology. 2017;152:1901-1914.e3.

27. Ashworth LA, Billett A, Mitchell P, Nuti F, Siegel C, Bousvaros A. Bousvaros A. Lymphoma risk in children and young adults with inflammatory bowel disease: analysis of a large single-center cohort. Inflamm Bowel Dis. 2012;18:838-843.

28. Virta LJ, Kolho K-L. Trends in early outpatient drug therapy in pediatric inflammatory bowel disease in Finland: a nationwide registerbased study in 1999-2009. ISRN Gastroenterol. 2012;2012:462642.

29. Chaparro M, Ramas M, Benítez JM, et al. Extracolonic cancer in inflammatory bowel disease: data from the GETECCU Eneida Registry. Am J Gastroenterol. 2017;112:1135-1143.

30. Ross SC, Strachan J, Russell RK, Wilson SL. Psychosocial functioning and health-related quality of life in paediatric inflammatory bowel disease. J Pediatr Gastroenterol Nutr. 2011;53:480-488.

31. Greenley RN, Hommel KA, Nebel J, et al. A meta-analytic review of the psychosocial adjustment of youth with inflammatory bowel disease. J Pediatr Psychol. 2010;35:857-869.

32. Aardoom MA, Linda Joosse ME, de Vries A, Levine A, de Ridder L. Malignancy and mortality in pediatric-onset inflammatory bowel disease: a systematic review. Inflamm Bowel Dis. 2018;24:732-741.

33. de Ridder L, Turner D, Wilson DC, et al. Malignancy and mortality in pediatric patients with inflammatory bowel disease. Inflamm Bowel Dis. 2014;20:291-300.

34. Jakobsen C, Bartek J, Wewer V, et al. Differences in phenotype and disease course in adult and paediatric inflammatory bowel disease - a population-based study. Aliment Pharmacol Ther. 2011;34:1217-1224.

35. Chaparro M, Garre A, Ricart E, et al. Differences between childhoodand adulthood-onset inflammatory bowel disease: the CAROUSEL study from GETECCU. Aliment Pharmacol Ther. 2019;49:419-428.

36. Van Limbergen J, Russell RK, Drummond HE, et al. Definition of phenotypic characteristics of childhood-onset inflammatory bowel disease. Gastroenterology. 2008;135:1114-1122.

\section{SUPPORTING INFORMATION}

Additional supporting information will be found online in the Supporting Information section at the end of the article.

How to cite this article: Malham M, Jakobsen C, Paerregaard A, Virta LJ, Kolho K-L, Wewer V. The incidence of cancer and mortality in paediatric onset inflammatory bowel disease in Denmark and Finland during a 23-year period: a populationbased study. Aliment Pharmacol Ther. 2019;00:1-7. https:// doi.org/10.1111/apt.15258 